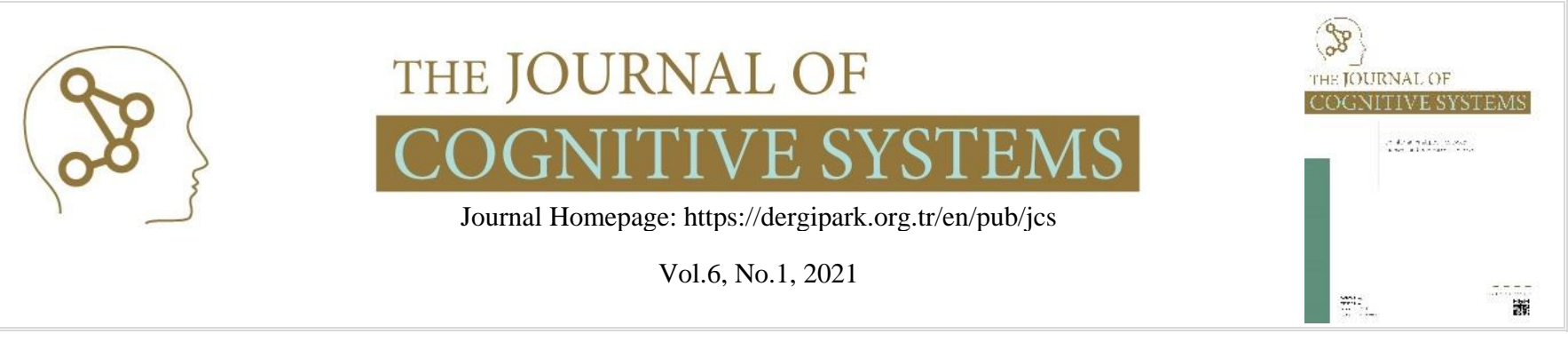

\title{
A Web-Based Software for Reporting Guidelines of Scientific Research
}

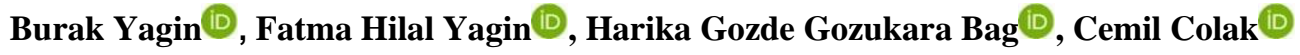 \\ Inonu University, Faculty of Medicine, Department of Biostatistics and Medical Informatics, Malatya, Turkey. (e-mail: \{burak.yagin, hilal.yagin, \\ harika.gozukara, cemil.colak\}@inonu.edu.tr
}

\section{ARTICLE INFO}

Received: March.,09.202

Revised: April,11.2021

Accepted: May.,19.2021

\section{Keywords:}

Epidemiological Research Designs

Research Reporting Guidelines

Python

Corresponding author: Fatma Hilal

Yagin

$\triangle$ hilal.yagin@inonu.edu.tr

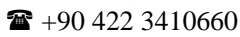

ISSN: 2548-0650

DOI: https://doi.org/10.52876/jcs.916182

\begin{abstract}
Aim: It is very important to use accurate reporting guidelines when reporting a study in cognitive science and health. This study aims to develop a web-based tool that leads to a reporting guideline that includes checklists and flowcharts for relevant research by type of research designs (qualitative, descriptive, experimental, and methodological studies, etc.)

Materials and Methods: The current study covers qualitative research, systematic review/metaanalysis, case presentations, case series, correlational (ecological), case-control, cross-sectional, cohort, randomized clinical trial, non-randomized clinical trial, field studies (for there are reporting guideline for primary protection measures), health care research (animal experiments, etc.), validity studies, consistency studies, simulation studies. For this purpose, the researcher is asked which epidemiological research design is useful and is directed to the reporting guideline for the relevant research type. During the development of the software, the DASH Library in the Python programming language was used.

Results: The Scientific Research Guidelines Software developed in this study can improve the reporting quality of the studies by guiding researchers to the correct reporting guide. The webbased software developed can be accessed at http://biostatapps.inonu.edu.tr/BAKY/. The software has English and Turkish language options.

Conclusion: Scientific Research Guidelines Software allows researchers to clearly state what they do and what they don't do in their study, how they do it, and what they find as a result. Besides, this software provides access to guides where they can learn about the meaning, strengths, and weaknesses of the study being done.
\end{abstract}

\section{INTRODUCTION}

$\mathrm{G}$ IVEN the large and growing volume of published articles, readers often find research reports that do not provide a clear and transparent explanation of methods and adequate reporting of results. If authors do not provide sufficient detail about the conduct of their work, they leave readers with an incomplete picture of the work done and its findings. Insufficiently reported studies can lead to misinterpretation and misapplication in clinical settings. It also provides misleading evidence that can be used to develop new research based on published studies. Therefore, funds allocated to support research may not be optimally used [1].

Since the early 1990s, research groups of major medical journal editors and content experts have developed reporting guidelines to help to improve the reporting quality of their articles in the healthcare field. Reporting guidelines are developed to report a particular type of epidemiological research. A reporting guideline line usually consists of a guiding checklist, flowchart, or clear text for authors. Carefully developed reporting guidelines provide authors with a minimal set of items that should be addressed when reporting a study. For example, PRISMA is a reporting guideline developed for Systematic reviews and MetaAnalysis. PRISMA was developed by a team of 29 people with meticulous work and includes a 27 -item checklist and a four-step flow chart. Initial reviews of reporting guidelines show that their use is associated with improved reporting quality [2].

Research reporting guidelines allow authors to properly write their research and give them the specific guidance they need during the reporting phase. Editors often advise authors to report on their work, but these recommendations tend to be vague and short, or too broadly long and daunting. For 
example, BMJ's recommendations for reporting are currently more than 20,000 words (http://resources.bmj.com/bmj/authors) $[2,3]$.

The development of more than 80 reporting guidelines to report different types of epidemiological investigations may result in uncertainty and misapplication. In addition, authors may not know where to access these guidelines and referees do not know how to use them. These guidelines contain recommendations that allow researchers to clearly say what they did and did not do in their study, how they did it, and what they found as a result of the research, thus allowing an honest discussion about the meaning of the study, its strengths and weaknesses $[4,5]$. Also, these guidelines often include a checklist of items that should be clearly communicated in the study and a flowchart showing what happened to the participants at each stage of the study [2].

The aim of this study is to develop a web-based tool that guidelines you to a reporting guideline that includes checklists and flow charts for the relevant research according to the research type (qualitative, descriptive, experimental, and methodological studies, etc.). Scientific research guidelines software can be accessed free of charge at http://biostatapps.inonu.edu.tr/BAKY/. It has two language options, English and Turkish.

\section{REPORTING RULES}

Reporting guidelines are very important for studies in cognitive science and health. Guidelines and checklists help individuals meet certain standards by providing a set of rules or principles that guidelines best behavior in a particular area. The World Health Organization submitted the checklist in 2008 and piloted its implementation in eight different hospitals around the world. The results of this study showed that the implementation of the checklist had a significant decrease in postoperative complication rates and surgical death. These results show that the implementation of the checklist can lead to significant improvements [6].

In recent years, many reporting guidelines have been developed. These guidelines usually contain the minimum information required for a complete and clear explanation of what was done and what was found during research, and specifically address issues that may have biased the research. Most internationally accepted reporting guidelines reflect the consensus of journal editors and experts in a particular field and also drawn on relevant evidence. Some journals already use these guidelines and require authors to report their work according to the relevant guidelines. Although studies evaluating the effects of using reporting guidelines on the quality of health research are still rare, studies have shown very promising results. However, to achieve an improvement in health research reporting globally, everyone involved in publishing research findings should have at least a basic knowledge of good research reporting principles and existing reporting guidelines. This applies not only to researchers and authors of research articles but also to journal editors and reviewers.

\subsubsection{Scientific Research Guidelines Software}

The Scientific Research manuals software was developed using the DASH Library in the Python programming language. The web-based software developed provides reporting guidelines for the following types of health research. The developed software is published at the website of https://biostatapps.inonu.edu.tr/.

\subsubsection{Qualitative Researches}

It is a type of research in which qualitative data collection methods such as observation, interview, and document analysis are used, and a process is followed to reveal perceptions and events realistically and holistically in the natural environment. The reporting guideline used in the software for qualitative research can be accessed at https://insights.ovid.com/pubmed?pmid=24979285 [7].

\subsubsection{Systematic Review/Meta-Analysis}

A systematic review, meta-analysis and meta-synthesis studies synthesize the results of research on the same subject and are research methods developed to summarize. While summarizing quantitative research results in meta-analyses Qualitative research results are summarized in metasyntheses. In systematic reviews, the results of many studies conducted by experts in the field with similar methods are synthesized. In order to perform a meta-analysis, a systematic review process must be done beforehand. However, when the findings of the studies included in the systematic review are not appropriate, meta-analysis is not performed [8]. In metasynthesis, the findings of qualitative research conducted in a specific field are evaluated and interpreted. New inferences are made by revealing similar and different aspects of these studies [9].

Different reporting guidelines should be used according to the types of systematic reviews, meta-analysis and metasynthesis studies. In systematic reviews and meta- analyses, "Preferred Reporting Items for Systematic Reviews and MetaAnalyzes (PRISMA)" is most frequently used [8, 10]. The reporting guideline used in the software for Systematic Reviews and Meta-Analyzes can be accessed http://www.prisma-statement.org/. In meta-synthesis studies, the quality of the research is generally evaluated with the "Critical Appraisal Skills Program (CASP) Tool as a Screening Tool" [11]. The reporting guideline used in the software for meta-synthesis studies can be https://casp-uk.bcdn.net/wp-content/uploads/2018/03/CASP-Qualitative-

Checklist-2018_fillable_form.pdf. When Cochrane metaanalysis is done from randomized controlled studies, "Cochrane Central Register of Controlled Trials" (CENTRAL) should be used [11,12]. The reporting guideline used in the software for Cochrane Meta-Analysis from Randomized Controlled Trials can be https://core.ac.uk/download/pdf/145317582.pdf. Quality of Reporting of Meta-analysis (QUOROM) is used in systematic reviews and meta-analyses of randomized controlled trials [13]. The reporting guideline used in the software for systematic reviews and meta-analyses of randomized controlled trials can be https://www.thelancet.com/pdfs/journals/lancet/PIIS0140673 699041495.pdf. Systematic Reviews and Meta-Analyzes of Observation Studies in Epidemiology (MOOSE) can be used for systematic reviews and meta-analysis of epidemiological observational studies [14]. The reporting guideline used in the software for Systematic Reviews and Meta-Analysis of Observational Studies can be https://www.ijo.in/documents/14MOOSE_SS.pdf. 
It is a report in which the findings, clinical course, and prognosis of a single case with an unexpected clinical disease are described, and previously reported cases are discussed, generally to indicate the place of the presented case in the general disease. The reporting guideline used in the software for Case Presentations Research is available at https://www.care-statement.org/checklist [15].

\subsubsection{Case Series}

It enables the identification of symptoms and findings and the definition of the case. It helps to better understand the spectrum and natural history of the disease. It is useful for clinical education. The reporting guideline used in the software for Case Series Research is available at https://www.neuropt.org/docs/default-source/research/csmcase-study-abstract-guidelines.pdf?sfvrsn=13325343_0 [16].

\subsubsection{Correlational (Ecological) Studies}

Ecological studies assess the overall disease frequency in a range of populations and look for a correlation with average exposure in populations. These studies are unique in that the analysis is not based on data on individuals. Rather, data points are average exposure levels and overall disease frequency in a range of populations. Therefore, the observation unit is not a person; instead, it is a whole population or group. The reporting guideline used in the software for Correlational (Ecological) Research can be accessed

https://www.ncbi.nlm.nih.gov/pmc/articles/PMC4902742/ [17].

\subsubsection{Case-Control Studies}

A study comparing people with a particular interest outcome ('cases') with people from the same population source but without that outcome, to examine the relationship between outcome and previous exposure (for example, an intervention). This design is particularly useful when the result is rare. The reporting guideline used in the software for Case-Control Research can be accessed at https://www.strobe-statement.org/index.php?id=availablechecklists [18].

\subsubsection{Cross-Sectional Studies}

These are the studies in which the prevalence of the disease of interest in the society is investigated, results are obtained generalizable to the whole society, and the whole society or a representative sample is examined. The reporting guideline used in the software for Cross-sectional Research can be accessed at https://www.strobestatement.org/index.php?id=available-checklists [18].

\subsubsection{Cohort Studies}

Cohort studies are analytical epidemiological studies planned to determine the relationship between a health problem and the reason thought to cause this health problem. The reporting guideline used in the software for Cohort Studies can be accessed at https://www.strobestatement.org/index.php?id=available-checklists [18].

\subsubsection{Randomized Clinical Trials}

It is a study design that randomly assigns individuals to an experimental group or a control group. While the study is being conducted, the only expected difference between the control and experimental groups in a randomized controlled study (RCT) is the study outcome variable. The reporting guideline used in the software for the Randomized Clinical Trial can be accessed at http://www.consortstatement.org/consort-2010 [19].

\subsubsection{Non-Randomized Clinical Trials}

It is a clinical trial in which participants are not assigned to different treatment groups by chance. Participants can choose the group they want to be included in, or they can be assigned to groups by researchers. The reporting guideline used in the software for Non-Randomized Clinical Trial can be accessed at https://www.cdc.gov/trendstatement/ [20].

\subsubsection{Field Studies (For Primary Protection Measures)}

Fieldwork is a research activity that takes place in the researcher context rather than an office or lab. The range of possible fieldwork methods and activities is very wide. Fieldwork also varies greatly in terms of how the researcher interacts (or does not) with the participants. The reporting guideline used in the software for Field Studies (For Primary Protection Measures) can be accessed at https://journals.plos.org/plosmedicine/article?id=10.1371/jou rnal.pmed.1000052 [21].

\subsubsection{Health Care Research (Animal Experiments etc.)}

Health research refers to many types of scientific research that aim to test ideas, answer questions, improve treatment options, and increase knowledge about human health. The reporting guideline used in the software for Health Care Research (Animal Experiments etc.) can be accessed at https://arriveguidelines.org/ [22,23].

\subsubsection{Validity Studies}

Indicates whether a scale measures what it aims to measure and its generalizability. It indicates that the phenomenon or judgment considered to be measured is measured correctly. A valid measurement means that the measuring tool is measuring the features it plans to measure. The reporting guideline used in the software for Validity Studies can be accessed at https://www.bmj.com/content/351/bmj.h5527 [24].

\subsubsection{Consistency Studies}

In Consistency Studies, when the measurements, observations, and examinations made in any research are repeated on the same people under the same conditions, the same observers will obtain the same results. If there is no observer variation, it is expected that the measurement results are theoretically the same or very close to each other in the examinations performed with the same method in two serum samples taken simultaneously from the same person. This same / similarity dimension is called the reliability/consistency of the observations. The reporting guideline used in the software for Consistency Studies can be 
accessed at https://www.bmj.com/content/351/bmj.h5527 [24].

\subsubsection{Simulation Studies}

Simulation studies are computer experiments that involve generating data by so-called random sampling. An important strength of simulation studies is the ability to understand the behaviour of statistical methods because some "real" (usually some parameters) are known from the data generation process. The reporting guideline used in the software for Simulation Studies can be accessed at https://www.cos.ufrj.br/uploadfile/1368206472.pdf [25].

\section{RESULTS}

The Scientific Research Guidelines Software developed in this study can improve the reporting quality of the studies by guiding researchers to the correct reporting guide. The web-based software developed can be accessed at http://biostatapps.inonu.edu.tr/BAKY/. The software has English and Turkish language options. The main and research designs menus of Scientific Research Guidelines Software are given in Figure 1.
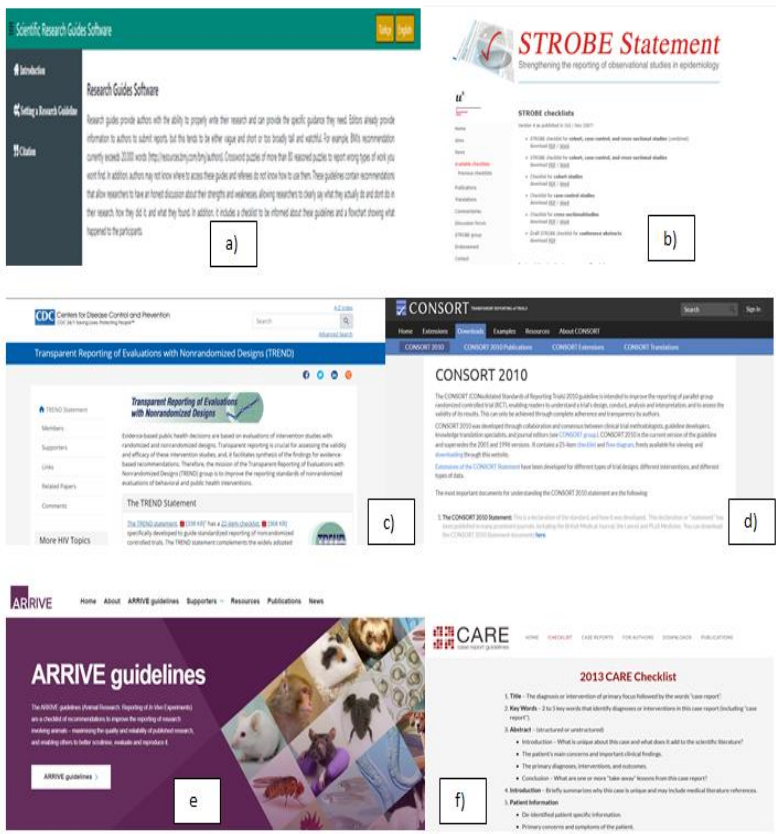

Fig.1. Main menus of Scientific Research Guidelines Software (a: Introduction menu; b: Reporting guidelines for Cohort, case-control, and cross-sectional studies - STROBE; c: Reporting guidelines for Nonrandomized designs - TREND; d: Reporting guidelines for Randomize designs - CONSORT; e: Reporting guidelines for Animal Experiments ARRIVE; f: Reporting guidelines for Case Report Studies - CARE.

\section{CONCLUSIONS}

The reliability and robustness of the medical research literature can be greatly improved by empowering authors, editors, and reviewers using tools that provide better reporting and improve the evaluation process. By using research reporting guidelines, clinicians, managers, and other healthcare professionals can make better evidence-based decisions, resulting in a much higher return on investment in health research [26].

More discipline should be taken when publishing research studies if new and effective research evidence/results are desired to improve health. Therefore, the way to provide more accurate and complete research reports is to adhere to scientific reporting rules [27].
It is the joint responsibility of all individuals and institutions involved in the financing, management, and publication of the research to report health research in a complete, accurate, open, and timely manner. Error-free and high-quality scientific research reports contribute to the more efficient use of the obtained research findings in clinical applications and allow the new scientific knowledge obtained and the progress of the care and treatment of patients [21].

Some of the benefits arising from the implementation of Scientific Research guidelines can be expressed as follows [23]:

1. Presentation quality and transparency of research increases,

2. Light up future research with good reporting is kept,

3. With advanced reporting, published research output may be maximized,

4. For the publication and reporting of research a common model is designed.

In addition to the benefits in designing and conducting research in the field of health, the reporting guideline set in the software can be used as an aid in the development of training courses in the design and application of research. The web-based scientific research guideline s software developed can make an important contribution to this process by facilitating global cooperation between researchers, higher education, and publishing communities.

\section{REFERENCES}

[1] Simera, I., Moher, D., Hoey, J., Schulz, K. F., \& Altman, D. G. (2010). A catalogue of reporting guidelines for health research. European journal of clinical investigation, 40(1), 35-53.

[2] Moher, D., Schulz, K. F., Simera, I., \& Altman, D. G. (2010). Guidance for developers of health research reporting guidelines. PLoS medicine, 7(2), e1000217.

[3] Altman, D. G., Simera, I., Hoey, J., Moher, D., \& Schulz, K. (2008). EQUATOR: reporting guidelines for health research. Open Medicine, 2(2), e49.

[4] Simera, I., Moher, D., Hirst, A., Hoey, J., Schulz, K. F., \& Altman, D. G. (2010). Transparent and accurate reporting increases reliability, utility, and impact of your research: reporting guidelines and the EQUATOR Network. BMC medicine, 8(1), 1-6.

[5] Stevens, A., Shamseer, L., Weinstein, E., Yazdi, F., Turner, L., Thielman, J., ... \& Moher, D. (2014). Relation of completeness of reporting of health research to journals' endorsement of reporting guidelines: systematic review. Bmj, 348 .

[6] Altman, D. G., \& Moher, D. (2005). Developing guidelines for reporting healthcare research: scientific rationale and procedures. Medicina clinica, 125, 8-13.

[7] Al-Busaidi, Z. Q. (2008). Qualitative research and its uses in health care. Sultan Qaboos University Medical Journal, 8(1), 11.

[8] Karaçam, Z. (2013). Sistematik derleme metodolojisi: Sistematik derleme hazırlamak için bir rehber. Dokuz Eylül Üniversitesi Hemșirelik Fakültesi Elektronik Dergisi, 6(1), 26-33.

[9] Polat, S., \& Osman, A. Y. (2016). Meta-sentez: Kavramsal bir çözümleme. Eğitimde Nitel Araştırmalar Dergisi, 4(2), 52-64.

[10] Tricco, A. C., Lillie, E., Zarin, W., O'Brien, K. K., Colquhoun, H., Levac, D., ... \& Straus, S. E. (2018). PRISMA extension for scoping reviews (PRISMA-ScR): checklist and explanation. Annals of internal medicine, 169(7), 467-473.

[11] Maribo, T., Jensen, C. M., Madsen, L. S., \& Handberg, C. (2020) Experiences with and perspectives on goal setting in spinal cord injury rehabilitation: a systematic review of qualitative studies. Spinal Cord, 58(9), 949-958.

[12] Kılıçkap, M. (2018). Meta-analizleri nasıl yorumlayalım: Türkiye'de kardiyovasküler risk faktörlerine yönelik yapılan meta-analizlerin metodolojik açıdan değerlendirilmesi. Turk Kardiyol Dern Ars, 46(7), 624-635.

[13] Moher, D., Cook, D. J., Eastwood, S., Olkin, I., Rennie, D., \& Stroup, D. F. (2000). Improving the quality of reports of meta-analyses of randomised controlled trials: the QUOROM statement. Oncology Research and Treatment, 23(6), 597-602. 
[14] Rajasekhar, A., Lottenberg, R., Lottenberg, L., Liu, H., \& Ang, D. (2011). Pulmonary embolism prophylaxis with inferior vena cava filters in trauma patients: a systematic review using the meta-analysis of observational studies in epidemiology (MOOSE) guidelines. Journal of thrombosis and thrombolysis, 32(1), 40-46.

[15] Agha, R. A., Fowler, A. J., Saeta, A., Barai, I., Rajmohan, S., Orgill, D. P., ... \& Rosin, D. (2016). The SCARE statement: consensus-based surgical case report guidelines. International Journal of Surgery, 34, 180-186.

[16] Zucker, D. M. (2009). How to do case study research. School of nursing faculty publication series, 2 .

[17] Morgan, R. L., Thayer, K. A., Bero, L., Bruce, N., Falck-Ytter, Y., Ghersi, D., ... \& Schünemann, H. J. (2016). GRADE: Assessing the quality of evidence in environmental and occupational health. Environment international, 92, 611-616.

[18] Cuschieri, S. (2019). The STROBE guidelines. Saudi journal of anaesthesia, 13(Suppl 1), S31.

[19] Moher, D., Schulz, K. F., \& Altman, D. (2001). The CONSORT statement: revised recommendations. JAMA, 285(15), 1987-1991.

[20] Treasure, E. (2004). The TREND statement. Evidence-based dentistry, 5(4), 88-91.

[21] Newton, P. N., Lee, S. J., Goodman, C., Fernández, F. M., Yeung, S., Phanouvong, S., ... \& White, N. J. (2009). Guidelines for field surveys of the quality of medicines: a proposal. PLoS medicine, 6(3), e1000052.

[22] Percie du Sert, N., Hurst, V., Ahluwalia, A., Alam, S., Avey, M. T., Baker, M., ... \& Würbel, H. (2020). The ARRIVE guidelines 2.0: Updated guidelines for reporting animal research. Journal of Cerebral Blood Flow \& Metabolism, 40(9), 1769-1777.

[23] Çolak, C., \& Parlakpınar, H. (2012). Hayvan deneyleri: in vivo denemelerin bildirimi: ARRIVE K1lavuzu-Derleme.

[24] Bossuyt, P. M., Reitsma, J. B., Bruns, D. E., Gatsonis, C. A., Glasziou, P. P., Irwig, L., ... \& Cohen, J. F. (2015). STARD 2015: an updated list of essential items for reporting diagnostic accuracy studies. Clinical chemistry, 61(12), 1446-1452.

[25] De França, B. N., \& Travassos, G. H. (2012). Reporting guidelines for simulation-based studies in software engineering.

[26] Erik von Elm, M. D., Altman, D. G., Egger, M., Pocock, S. J., Gøtzsche, P. C., \& Vandenbroucke, J. P. (2007). The Strengthening the Reporting of Observational Studies in Epidemiology (STROBE) statement: guidelines for reporting observational studies. Ann Intern Med, 147(8), $573 \mathrm{e} 7$.

[27] Liberati, A., Altman, D. G., Tetzlaff, J., Mulrow, C., Gøtzsche, P. C., Ioannidis, J. P., ... \& Moher, D. (2009). The PRISMA statement for reporting systematic reviews and meta-analyses of studies that evaluate health care interventions: explanation and elaboration. Journal of clinical epidemiology, 62(10), e1-e34.

\section{BIOGRAPHIES}

Burak Yağın obtained his BSc. degree in Statistics from Gazi University in 2017. He currently continues his MSc. education in biostatistics and medical informatics from Inonu University. His research interests are cognitive systems, data mining, machine learning, deep learning, and image processing.

Fatma Hial Yağın obtained her BSc. degree in Statistics from Gazi University in 2017. She received MSc. degree in biostatistics and medical informatics from Inonu University in 2020. She currently continues her $\mathrm{Ph} . \mathrm{D}$. education in biostatistics and medical informatics from Inonu University. In 2019, she joined the Department of Biostatistics and Medical Informatics at Inonu University as a researcher assistant. Her research interests are cognitive systems, data mining, machine learning, deep learning, and image processing.

Harika Gözde Gözükara Bağ obtained her BSc. degree in Agricultural Engineering from Ankara University in 2000. She received MSc. degree in Biostatistics from the Inonu University in 2004, and a PhD degree in Biostatistics from Hacettepe University in 2011. She is currently working as an Associate Professor at the Department of Biostatistics and Medical Informatics at Inonu University since 2019. She is active in teaching and her research interests are genetics, survival analysis and meta-analysis.

Cemil Colak obtained his BSc. degree in Statistics from Ondokuz Mayıs University in 1999. He received MSc. degree in Biostatistics from the Inonu University in 2001, and a PhD degree in the Graduate Department of Biostatistics and Medical Informatics of Ankara University in 2005. His research interests are cognitive systems, data mining, reliability, and biomedical system, genetics, and bioengineering. In 2016, he joined the Department of Biostatistics and Medical Informatics at Inonu University as a Professor, where he is presently a professor. He is active in teaching and research in general image processing, artificial intelligence, data mining, and analysis. 